\title{
ON REPRESENTATIONS OF THE SYMMETRIC
}

GROUPS, NILPOTENT MATRICES, SYSTEMS,

VECTORBUNDLES AND SCHUBERT CELLS.

Michie1 Hazewinke 1

Dept. Math., Erasmus Univ. Rotterdam

P.0. Box 1738, Rotterdam,

The Netherlands

Introduction. Let $k$ be a partition of $n, k_{1}=\left(\kappa_{1}, \ldots, \kappa_{m}\right), \kappa_{1} \geq \ldots \geq k_{m} \geq 0$, $\Sigma \kappa_{i}=n$. We identify partitions $\left(k_{1}, \ldots, k_{m}\right)$ and $\left(k_{1}, \ldots, k_{m}, 0, \ldots, 0\right)$. One defines a partial order on the set of all partitions as follows

$$
\left(\kappa_{1}, \ldots, \kappa_{m}\right)>\left(k_{1}^{\prime}, \ldots, k_{m}^{\prime}\right) \Leftrightarrow \sum_{i=1}^{r} \kappa_{i} \leq \sum_{i=1}^{r} k_{i}^{\prime}, r=1, \ldots, m
$$

Thus for example $(2,2,1)>(3,2)$. If $K>K^{\prime}$ we say that $K$ specializes to $K^{\prime}$ or that $K$ is more general than $K^{\prime}$. The reverse order has been called the dominance order. It occurs naturally in several seemingly rather unrelated parts of pure and applied mathematics. Some of these occurrences can be labelled by the words and phrases

(i) Snapper conjecture (on the representations of symetric groups)

(ii) Gale Ryser theorem (on existence of $(0,1)$-matrices)

(iii) Muirheads inequality (a symmetric mean inequality)

(iv) Gerstenhaber-Hesselink theorem (on orbit closure properties of $\mathrm{SL}_{\mathrm{n}}$ acting on nilpotent matrices)

(v) Kronecker indices (on the orbit closure, or degeneration, properties of linear control systems acted on by the socalled feedback group)

(vi) Double stochastic matrices (when is a partition "an average" of another partition)

(vii) Shatz's theorem (on degeneration of vectorbundles (over the Riemann sphere))

These will be described in more detail in section 2 below.

In addition the same ordening plays a considerable role in theoretical chemistry in the theory of chiral molecules, i.e. molecules that are optically active $[11,16,18]$.

Certain of these manifestations of this specialization order are known to be intimatedly related. Thus (i), (ii), (iii) and (vi) are very much related $[2,13]$ and so are (v) and (vii) [15]. This talk is a report of work done jointly with Clyde Martin of Case Western Reserve Univ, which shows that all these manisfestations of this order are intimately related and that their common meeting ground, so to speak, seems to be the ordexing defined by closure relations of the SchubertCells (with respect to a standard basis) of a Grassmann manifold. I.e. a Schubert- 
cell $\operatorname{sC}(\lambda)$ is more general than $\operatorname{SC}\left(\lambda^{\prime}\right)$; in symbols: $\operatorname{sC}(\lambda)>\operatorname{sC}\left(\lambda^{\prime}\right)$, iff $\overline{\operatorname{SC}(\lambda)} \supset \mathrm{SC}\left(\lambda^{\prime}\right)$. This order in turn is much related to the Bernstein-GelfandGelfand ordening on the Weyl group $S_{n}$. It is in fact the quotient ordering induced by the canonical map of the manifold of all flags in $\mathbb{R}^{n+m}$ to the Grassmann manifold of $n$-planes in $(n+m)$-space. Full details will appear elsewhere [8].

\section{SEVERAL MANIFESTATIONS OF THE SPECIALIZATION ORDER}

2.1. The Snapper conjecture. Let $k=\left(\kappa_{1}, \ldots, \kappa_{m}\right)$ be a partition of $n$. Let $s_{K}$ be the corresponding Young subgroup $S_{K}=s_{K_{1}} \times \ldots \times S_{K_{m}}$, where $s_{K_{i}}$ is seen as the subgroup of $\mathrm{s}_{\mathrm{n}}$ acting on the letters $k_{1}+\ldots+k_{i-1}+1, \ldots, k_{1}+\ldots+k_{i}$. (If $k_{m}=0$ the factor $S_{K_{m}}$ is deleted). Take the trivial representation of $S_{K}$ and induce this up to $s_{n}$. Let $\rho(k)$ denote the resulting representation. It is of dimension $n ! / k_{1} ! \ldots k_{m}$ ! and it can be easily described as follows. Take $m$ symbols $a_{1}, \ldots, a_{m}$ and considex all associative (but noncommutative) words $\varepsilon_{1} \ldots \varepsilon_{n}$ of length $n$ in the symbols $a_{1}, \ldots, a_{m}$ such that $a_{i}$ occurs precisely $k_{i}$ times. Let $w\left(\kappa_{1}, \ldots, \kappa_{m}\right)=W(k)$ denote this set. Then $S_{n}$ acts on $W(K)$ by $\sigma\left(\varepsilon_{1} \ldots \varepsilon_{n}\right)=\varepsilon_{\sigma(1)} \varepsilon_{\sigma(2)} \cdots \varepsilon_{\sigma(n)}$. Let $V(K)$ be the vectorspace with the elements of $W(K)$ as basis vectors. Extending the action of $\mathrm{S}_{\mathrm{n}}$ linearly this gives a representation of $\mathrm{S}_{\mathrm{n}}$ and this is the representation $\rho(K)$.

Now the irreducible representations of $\mathrm{S}_{\mathrm{n}}$ are also labelled by partitions. Let [K] be the irreducible representation belonging to the partition $K$. Snapper [21] proved that $[K]$ occurs in $\rho\left(K^{\prime}\right)$ only if $K<K^{\prime}$ and conjectured the reverse implication. Liebler and Vitale [14] proved that $\kappa^{\prime}<K^{\prime} \Rightarrow \rho(K)$ is a direct summand of $\rho\left(K^{\prime}\right)$ which of course implies that $k<k^{\prime} \Rightarrow[K]$ occurs in $\rho\left(K^{\prime}\right)$. Another proof of this implication (via a different generalization) was given by Lam [13].

2.2. The Gale-Ryser theorem $([5,19])$. Let $\mu$ and $\nu$ be two partitions of $n$. Then there is a matrix consisting of zero's and one's whose columns sum to $\mu$ and whose rows sum to $\nu$ iff $\nu>\mu^{*}$. Here $\mu^{*}$ is the dual partition of $\mu$ defined by $\mu_{i}^{*}=\left\{j \mid \mu_{j} \geq i\right\}$. (If $S$ is a set then $S$ stands for the number of elements in that set.) For example $(2,2,1)^{*}=(3,2)$.

2.3. Doubly stochastic matrices. A matrix $M=\left(m_{i j}\right)$ is called doubly stochastic if $m_{i j} \geq 0$ for all $i, j$ and if all the columns and all the rows add up to 1 . Let $\mu$ and $\nu$ be two partitions of $n$. One says that $\mu$ is an average of $\nu$ if there is a doubly stochastic matrix $M$ such that $\mu=M v$. Then there is the theorem that $\mu$ is an average of $\nu$.iff $\mu>\nu$ (in the specialization order).

2.4. Muirhead's inequality. One of the best-known inequalities is $\left(x_{1} \ldots x_{n}\right)^{1 / n_{\leq}}$ $n^{-1}\left(x_{1}+\ldots+x_{n}\right)$. A far-reaching generalization due to Muirhead [22] goes as follows. Given a vector $p=\left(p_{1}, \ldots, p_{n}\right), p_{i} \geq 0$ one defines a symmetrical mean (of the nonnegative variables $x_{1}, \ldots, x_{n}$ ) by the formula 


$$
[p](x)=(n !)^{-1} \sum_{\sigma} x_{1}^{p_{\sigma(1)}} \ldots x_{n}^{p_{\sigma(n)}}
$$

where the sum runs over all permutations $\sigma \in \mathrm{S}_{\mathrm{n}}$. Then one has Muirhead's inequality which states that $[p](x) \leq[q](x)$ for all non-negative values of the variables $x_{1}, \ldots, x_{n}$ iff $p$ is an average of $q$, so that in case $p$ and $q$ are partitions of $n$ this happens iff $\mathrm{p}>\mathrm{q}$. The geometric mean - arithmetic mean inequality thus arises from the specialization relation $(1, \ldots, 1)>(n, 0, \ldots, 0)$.

2.6. Completely reachable systems. Let $L_{m, n}$ denote the space of all pairs of real matrices $(A, B)$ of sizes $n \times n$ and $n \times m$ respectively. To such a pair ( $A, B$ ) one associates a control system given by the differential equations

$$
\dot{x}=A x+B u, x \in \mathbb{R}^{n}, u \in \mathbb{R}^{m}
$$

where the u's are the inputs or controls. The pair (A,B), or equivalently, the system (2.7), is said to be completely reachable if the reachability matrix $R(A, B)=\left(B: A B: \ldots: A^{n} B\right)$ consisting of the $(n+1)(n x m)$-blocks $A^{i} B, i=0, \ldots, n$ has maximal rank. $n$. In system theoretic terms this is equivalent to the property that for any two points $x, x^{\prime} \in \mathbb{R}^{n}$ one can steer $x(t)$ to $x^{\prime}$ in finite time starting from $x(0)=x$ by means of suitable control functions $u(t)$. Let $L_{m, n}^{c r}$ denote the space of all completely reachable pairs of matrices $(A, B)$. The Lie-group $F$ of all block lower diagonal matrices $\left(\begin{array}{ll}\mathrm{S} & 0 \\ \mathrm{~T}\end{array}\right), \mathrm{S} \in \mathrm{GL}_{\mathrm{n}}(\mathbb{R}), \mathrm{T} \in \mathrm{GL}_{\mathrm{m}}(\mathbb{R})$, $\mathrm{K}$ an $\mathrm{m} \times \mathrm{n}$ matrix, acts on $\mathrm{L}_{\mathrm{m}, \mathrm{n}}^{\mathrm{cr}}$ by according to the formula

$$
(A, B)^{g}=\left(\mathrm{SAS}^{-1}+\mathrm{SBTS}^{-1} \mathrm{~K}, \mathrm{SBT}\right), \mathrm{g}=\left(\begin{array}{ll}
\mathrm{S} & 0 \\
\mathrm{~K} & \mathrm{~T}
\end{array}\right)
$$

The 'generating transformations' $(A, B) \mapsto\left(S A S^{-1}, S B\right)$ (base change in state space), $(A, B) \mapsto(A, B T)$ (base change in input space) and $(A, B) \mapsto(A+B K, B)$ (state space feedback), occur naturally in design problems (of control loops) in electrical engineering. It is now a theorem of Kalman [10] that the orbits of $F$ acting on $\mathrm{L}_{\mathrm{m}, \mathrm{n}}^{\mathrm{cr}}$ correspond bijectively with partitions of $\mathrm{n}$. The partition belonging to $(A, B) \in L_{m, n}^{c r}$ is found as follows. Let $d_{j}$ be the dimension of the subspace of $\mathbb{R}^{n}$ spanned by the vectors $A^{i} b_{r}, r=1, \ldots, m, i \leq j$ where $b_{r}$ is the $r$-th column of $B$. Let $e_{j}=d_{j}-d_{j-1}, d_{-1}=0$ then the partition corresponding to $(A, B)$ is the dual partition of $\left(e_{0}, e_{1}, e_{2}, \ldots, e_{n}\right)$, i.e. $k(A, B)=\left(e_{0}, e_{1}, \ldots, e_{n}\right) *$. The numbers $k_{1} \geq \ldots \geq k_{m}$ making up $k(A, B)$ are called the Kronecker indices of $(A, B)$. (Because the problem of classifying pairs $(A, B)$ up to feedback equivalence, $i$.e. up to the action of $F$, is a subproblem of the problem of classifying pencils of matrices studied by Kronecker: to $(A, B)$ one associates the pencil (A-sI!B)). 
Let $\Theta_{K}$ be the orbit of $F$ acting on $L_{m, n}^{c r}$ labelled by $K$. Then a second theorem, noted by a fair number of people independently of each other (Kalman, Hazewinke1, Byrnes, Martin,...), but never yet published, states that $\theta_{K} \supset \theta_{K^{\prime}} \Leftrightarrow K>K$. In control theoretic terms this theorem says something about degeneration of systems or system failure.

2.9. Vectorbundles over the Riemann sphere. Let $\mathrm{E}$ be a holomorphic vectorbundle over the Riemann sphere $S^{2}=\mathbb{P}^{1}(\mathbb{C})$. Then according to Grothendieck [4] E splits as a direct sum of line bundles

$$
E \simeq L\left(k_{1}\right) \oplus \ldots \oplus L\left(\kappa_{m}\right)
$$

where $L(i)$ is the unique (up to isomorphism) line bundle over $\mathbb{P}^{1}(\mathbb{T})$ of degree $i, L(i)=L(1) i, i \in \mathbb{Z}$, where $L(1)$ is the canonical very ample line bundle of $\mathbb{P}^{1}(\mathbb{C})$. Thus each holomorphic vectorbundle $E$ over $\mathbb{P}^{1}(\mathbb{C})$ defines an $m$-tuple of integers $K(E)$ (in decreasing order). The bundle $E$ is called positive if $k_{i}(E) \geq 0$ for all $i=1, \ldots, m$. Concerning these positive bundles there is now the following degeneration result of Shatz [20]. Let $E_{t}$ be a holomorphic family of m-dimensional vectorbundles over $\mathbb{P}^{1}(\mathbb{C})$. Then for all small enough $t, k\left(E_{t}\right)>k\left(E_{o}\right)$. And inversely if $k>K^{\prime}$ then there is a homorphic family $E_{t}$ such that $k\left(E_{t}\right)=k$ for $t$ small $t \neq 0$ and $k\left(E_{0}\right)=K^{\prime}$.

2.11. Orbits of nilpotent matrices. Let $\mathrm{N}_{\mathrm{n}}$ be the space of all $\mathrm{n} \times \mathrm{n}$ complex nilpotent matrices. Consider $\mathrm{SL}_{\mathrm{n}}(\mathbb{C})$ or $\mathrm{GL}_{\mathrm{n}}(\mathbb{C})$ acting on $\mathrm{N}_{\mathrm{n}}$ by similarity, i.e. $A^{S}=\operatorname{SAS}^{-1}\left(A \in N_{n}, S \in G L_{n}(\mathbb{C})\right)$. By the Jordan normal form theorem the orbits of this action are labelled by partitions of $n$. Let $(K)$ be the orbit consisting of all nilpotent matrices similar to the one consisting of the Jordan blocks $J\left(\kappa_{i}\right)$, $i=1, \ldots$, m where $J\left(\kappa_{i}\right)$ is the $k_{i} \times k_{i}$ matrix with 1 's just above the diagonal and zero's everywhere else. Then the Gerstenhaber - Hesselink theorem says that $O(K) \supset O\left(K^{\prime}\right)$ iff $K<K^{\prime}$. (Note the reversion of the order with respect to the result on orbits described in 2.6 above.)

2.12. A schematic overview of the various relations between all these manifestations of the specialization order can be found in section 5 below.

\section{GRASSMANN MANIFOLDS AND CLASSIFYING VECTOR BUNDLES}

Before outlining how the various manifestations of the specialization order are connected to each other we need to define Grassmann manifolds, the classifying vectorbundle over them and their Schubert cell decomposition (in section 4 below).

3.1. Grassmann manifolds. Fix two numbers $m, n \in \mathbb{N}$. Then the Grassmann manifold $G_{n}\left(\mathbb{C}^{n+m}\right)$ consists of all $n$-dimensional subspaces of $\mathbb{c}^{n+m}$. Thus for example $G_{1}\left(\mathbb{c}^{m+1}\right)$ is the $m$ dimensional complex projective space $\mathbf{P}^{\mathrm{m}}(\mathbb{C})$. Let $\mathbb{d}_{\text {reg }}^{\mathrm{n} \times(\mathrm{n}+\mathrm{m})}$ be the space of 
all complex $n \times(n+m)$ matrices of rank $n$. Let GL $(\mathbb{C})$ act on this space by multiplication on the left. Then the, quotient space $\mathbb{C}^{n \times(n+m)} / G L_{n}(\mathbb{C})$ is $G_{n}\left(\mathbb{C}^{n+m}\right)$. The identification is done by associating to $M \in \mathbb{d}_{r e g}^{n \times(n+m)}$ the subspace of $\mathbb{d}^{n+m}$ generated by the rows of $M$.

$G_{n}\left(\mathbb{C}^{n+m}\right)$ inherits a natural holomorphic manifold structure from $\mathbb{d}^{n \times(n+m)}$. For a detailed description of $G_{n}\left(\mathbb{C}^{n+m}\right)$ cf. e.g. [17]

3.2. The classifying bundle. We define a holomorphic vector bundle $\xi_{\mathrm{m}}$ over $G_{n}\left(\mathbb{C}^{n+m}\right)$ as follows. For each $x$ let the fibre over $x, \xi_{m}(x)$, be the quotient space $\mathbb{C}^{n+m} / x$. More precisely define the bundle $n_{n}$ over $G_{n}\left(\mathbb{C}^{n+m}\right)$ by

$$
n_{n}=\left\{(x, v) \in G_{n}\left(\mathbb{c}^{n+m}\right) \times \mathbb{a}^{n+m} \mid v \in x\right\}
$$

with the obvious projection $(x, v) \quad x$. Then $\xi_{m}$ is the quotient bundle of the trivial vector bundle $G_{n}\left(\mathbb{C}^{n+m}\right) \times \mathbb{C}^{n+m}$ by $\eta_{n}$. Both $\xi_{m}$ and $\eta_{n}$ can be used as universal or classifying bundles (cf. [17] for $n_{n}$ as a universal bundle). Let $E$ be an m-dimensional vector bundle over a complex analytic manifold $\Pi$. Let $\Gamma(E)=\Gamma(E, \Pi)$ be the space of all holomorphic sections of $E$, i.c. the space of all holomorphic maps $s: \Pi \rightarrow E$ such that $p \circ s=i d$, where $p: E \rightarrow \Pi$ is the bundle projection. The universality, or classifying, property of $\xi_{\mathrm{m}}$ in the setting of complex analytic manifolds now takes the following form. Suppose $V \subset \Gamma(E)$ is an $(n+m)$-dimensional subspace such that for each $x \in \Pi$ the vectors $s(x), s \in V$ span $E(x)$, the fibre of $E$ over $x$. Now identy $V \simeq \mathbb{C}^{n+m}$ and associate to $x \in \Pi$ the point of $G_{n}\left(\mathbb{C}^{n+m}\right)$ represented by $\operatorname{Ker}(V \rightarrow E(x))$. This gives a holomorphic map $\psi_{E}: I \rightarrow G_{n}\left(\mathbb{C}^{n+m}\right)$ such that the pul1back of $\xi_{m}$ by means of $\psi_{E}$ is isomorphic to $E, \psi_{E}^{\prime} \xi_{m} \simeq E$. It is universality properties such as this one which account for the importance of the bundles $\xi_{m}$ and/or $\eta_{n}$ in differential and algebraic topology [17], algebraic geometry and also system and control theory (cf. [24] for the last mentioned).

The bundle $\xi_{\mathrm{m}}$ has a number of obvious holomorphic sections, viz. the sections defined by $\varepsilon_{i}(x)=e_{i} \bmod x$ where $e_{i}$ is the $i-t h$ standard basis vector of $\mathbb{c}^{n+m}$, $i=1, \ldots, m$. And, as a matter of fact, it is not difficult to show that $\Gamma\left(\xi_{m}, G_{n}\left(\mathbb{C}^{n+m}\right)\right)$ is $(n+m)$-dimensional and that the $\varepsilon_{1}, \ldots, \varepsilon_{n}$ from a basis for this space of holomorphic sections.

\section{SCHUBERT CELLS}

4.1. Schubert cells. Consider again the Grassmann manifold $G_{n}\left(\mathbb{C}^{m+n}\right)$. Let $\underline{A}=\left(A_{1}, \ldots, A_{n}\right)$ be a sequence of $n$-subspaces of $\mathbb{C}^{n+m}$ such that $0 \not A_{1} \subset A_{2} \subset \ldots \underset{f}{\subset} \ldots A_{n}$.
To each such sequence $\underline{A}$ we associate the closed subset

$$
\operatorname{SC}(\underline{A})=\left\{x \in G_{n}\left(\mathbb{C}^{m+n}\right) \mid \operatorname{dim}\left(x \cap A_{i}\right) \geq i\right\}
$$


and call it the closed Schubert-cell of the sequence $\underline{A}$. In particular if $0<\lambda_{1}<\lambda_{2}<\ldots<\lambda_{n} \leq n+m$ is a strictly increasing sequence of natural numbers $\leq \mathrm{n}+\mathrm{m}$ then we define (setting $\lambda=\left(\lambda_{1}, \ldots, \lambda_{n}\right)$ )

$$
\operatorname{sc}(\lambda)=\operatorname{sc}\left(\mathbb{c}^{\lambda}, \ldots, \mathbb{d}^{\lambda} n_{1}\right)
$$

where $\mathbb{C}^{r}$ is viewed as the subspace of all vectors in $\mathbb{C}^{n+m}$ whose last $n+m-r$ coordinates are zero.

4.4. Flag manifolds and the Bruhat decomposition. A $\underline{\text { flag }}$ in $\mathbb{a}^{\mathrm{n}+\mathrm{m}}$ is a sequence of subspaces $\underline{F}=F_{1} \subset \ldots \subset F_{n+m} \subset \mathbb{C}^{n+m}$ such that $\operatorname{dim} F_{i}=i$. Let $F \ell\left(\mathbb{C}^{n+m}\right)$ denote the analytic manifold of all flags in $\mathbb{c}^{\mathrm{n}+\mathrm{m}}$. There is a natural holomorphic mapping $F \ell\left(\mathbb{C}^{n+m}\right) \rightarrow G_{n}\left(\mathbb{C}^{n+m}\right)$ given by asspciating to a flag $\underline{F}$ its $n$-th element $F_{n}$. The $f l a g$ manifold can be seen as the space of all cosets $B g, g \in G_{n+m}(\mathbb{C})$ where $B$ is the Bore1 subgroup of all lower triangular matrices in $\mathrm{GL}_{\mathrm{n}+\mathrm{m}}(\mathbb{C})$. The mapping $\mathrm{GL}_{\mathrm{n}+\mathrm{m}}(\mathbb{C}) \rightarrow$ $F l\left(\mathbb{C}^{n+m}\right)$ associates to a matrix $g$ the $f l a g \underline{F}(g)$ whose $i$-th element is the subspace of $\mathbb{c}^{\mathrm{n}+\mathrm{m}}$ spanned by the first in row vectors of $\mathrm{g}$.

Now view $\mathrm{S}_{\mathrm{n}+\mathrm{m}}$, the symmetric group on $\mathrm{n}+\mathrm{m}$ letters as a subgroup of $\mathrm{GL}_{\mathrm{n}+\mathrm{m}}(\mathbb{C})$ by letting it permute the basis vectors $\left(\sigma\left(\rho_{i}\right)=\rho_{\sigma(i)}\right)$. Then in $G L_{n+m}(\mathbb{C})$ we have the socalled Bruhat decomposition

$$
\mathrm{GL}_{\mathrm{n}+\mathrm{m}}(\mathbb{C})=\bigcup_{\sigma}^{\cup} B \sigma B \quad \text { (disjoint union) }
$$

Where $\sigma$ runs through the Weyl group $S_{n+m}$ of $\mathrm{GL}_{n+m}(\mathbb{C})$. An analogous decomposition holds in a considerable more general setting (reductive groups, cf. [25], section 28).

4.6. The Bernstein-Gelfand-Gelfand order. The closure of a double coset BoB is necessarily a union of other double cosets (by continuity). This defines an ordering on the Weyl group $\mathrm{S}_{\mathrm{n}+\mathrm{m}}$ defined by

$$
\sigma>\tau \Leftrightarrow \overline{\mathrm{B} \sigma \mathrm{B}} \supset \mathrm{B} \tau \mathrm{B}
$$

This ordering plays a considerable role in the study of cohomology of flag spaces [1] and also in the theory of highest weight representations [27, 26].

Let $\mathrm{H}$ be the subgroup of $\mathrm{S}_{\mathrm{n}+\mathrm{m}}$ consisting of all block lower triangular matrices of the form $\left(\begin{array}{lc}s_{11} & 0 \\ s_{21} & S_{22}\end{array}\right), s_{11} \in G_{n}(\mathbb{C}), s_{22} \in G_{m}(\mathbb{C}), s_{21}$ and arbitrary $m \times n m a t r i x$. Then, using the remarks made in subsection 4.4 above, one sees that $G_{n}\left(\mathbb{C}^{n+m}\right)$ is the coset space $\left\{\mathrm{Hg} \mid \mathrm{g} \in \mathrm{GL}_{\mathrm{n}+\mathrm{m}}(\mathbb{C})\right\}$. Now let $\sigma \in \mathrm{S}_{\mathrm{n}+\mathrm{m}}$ and let $\lambda_{1}<\ldots<\lambda_{\mathrm{n}}$ be the $\mathrm{n}$ natural numbers in increasing order determined by $\sigma\left(e_{\lambda_{i}}\right) \in\left\{e_{1}, \ldots, e_{n}\right\}, i=1, \ldots, n$. Then one easily sees that the image of $B \circ B$ undèr $G_{n+m}(\mathbb{C}) \rightarrow G_{n}\left(\mathbb{C}^{n+m}\right)$, i.e. the set of all spaces spanned by matrices of the form $h \circ b, h \in H, b \in B$ is the open schubert 
cell of all elements in $G_{n}\left(\mathbb{C}^{n+m}\right)$ spanned by the rows of a matrix of the form

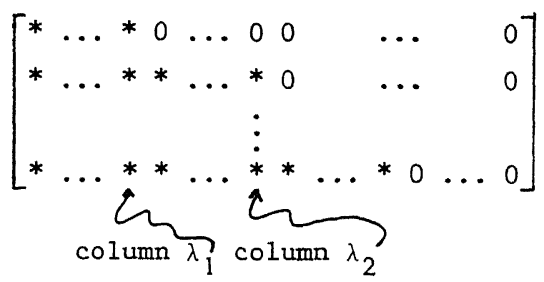

where the last * in each row is nonzero. The closure of this open Schubert-cell is the Schubert-cell $\operatorname{SC}(\lambda)$ defined in (4.3) above.

One easily checks that

$$
\operatorname{SC}(\mu) \subset \operatorname{SC}(\lambda) \Leftrightarrow \mu_{i} \leq \lambda_{i}, i=1, \ldots, n
$$

and this order on the Schubert cells $\operatorname{SC}(\lambda)$, or the equivalent ordening on $n-$ tuples of natural numbers, is therefore a quotient of the BGG order on the Weyl group $\mathrm{S}_{\mathrm{n}+\mathrm{m}}$. It is the induced order on the set of $\operatorname{cosets}\left(\mathrm{s}_{\mathrm{n}} \times \mathrm{s}_{\mathrm{m}}\right) \sigma, \sigma \in \mathrm{S}_{\mathrm{n}+\mathrm{m}}$. (Obviously if $\tau \in S_{n} \times S_{m}$, then $\tau \sigma\left(e_{\lambda_{i}}\right) \in\left\{e_{1}, \ldots, e_{n}\right\}$ if $\left.\sigma\left(e_{\lambda_{i}}\right) \in\left\{e_{1}, \ldots, e_{n}\right\}\right)$.

(And inversely the Weyl order is determined by the associated orders of Schubert cells in the sense that $\sigma>\tau$ in $S_{n}$ iff for all $k=1, \ldots, n-1$ we have for the associated Schubert cells in $G_{k}\left(\mathbb{C}^{n}\right)$ that $S C(\sigma) \subset S C(\tau)$; this is a rather efficient way of calculating the Weyl order).

\section{INTERRELATIONS}

Now that we have defined the concepts we need we can start to describe some interrelations between the various manifestations of the specialization order we discussed in section 2 above.

5.1. Overview of the various relations. A schematic overview of the various interconnections is given by the following diagram. In this diagram we have put together in boxes the manifestations which are more or less known to be intimatedly related and have explicitly indicated the new relations to be discussed in detail below.

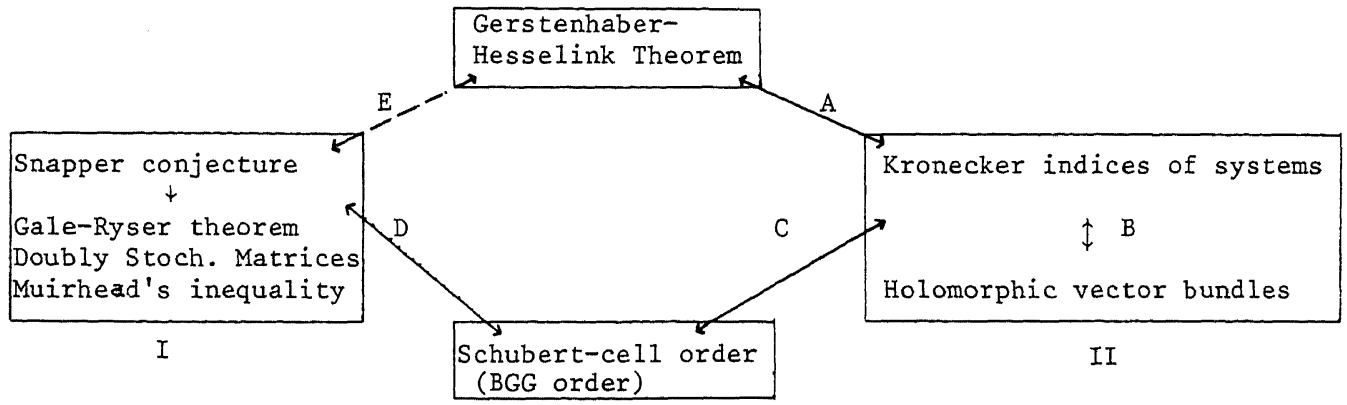


5.2. On the various relations. The manifestations of the specialization order in box I are well known to be intimatedly related $[2,5,11,13]$. Very much related is also the Ruch - Schönhofer theorem [18] which states that $<\rho(\kappa), \bar{\rho}(\mu)>\geq 1$ iff $\kappa>\mu^{*}$ where $<$, > denotes the usual innerproduct (which counts how many irreducible representations there are in common), and where $\bar{\rho}(\mu)$ is the representation of $S_{n}$ obtained by inducing the alternating representation of $\mathrm{S}_{\mu}$. The link between this theorem and the Gale - Ryser theorem are given by Mackey's intertwining number theorem [29], \$44 and Coleman's characterization [28] of double costs of Young subgroups.

Relation $A$ in the diagram is essentially established by giving two virtually identical proofs of the two theorems and these results can then be used to give natural continuous isomorphisms between feed-back orbits of systems and similarity orbits of nilpotent matrices. More details are in section 7 below. For connection $B$ one associates to a system $\Sigma \in L_{m, n}^{c r}$ a vector bundle $E(\Sigma)$ of dimension m over $\mathbb{P}^{1}(\mathbb{C})$, The construction used is a modification of the one in [15]. It has the advantage that one sees immediately that $\kappa(\Sigma)=\kappa(E(\Sigma))$. For connection $C$ one uses the classifying morphism $\psi_{E}: \mathbb{P}^{1}(\mathbb{C}) \rightarrow G_{n}\left(\mathbb{C}^{n+m}\right)$ attached to a positive bundle $E$ over $\mathbb{P}^{1}(\mathbb{C})$ (cf. section 3.2 above); It turns out that the invariants of $E$ can be recovered from $\psi_{E}$ be considering the dimensions of the spaces $A_{1}, \ldots, A_{n}$ such that Im $\psi_{E} \subset S C(\underline{A})$. To establish a link between representations of $S_{n+m}$ and Schubertcells we construct a family of representations of $S_{n+m}$ parametrized by $G_{n}\left(\mathbb{C}^{n+m}\right)$, which can be used to give a deformation type proof of the Snapper cnnjecture (in the Liebler-Vitale form) cf. section 11 below. This is not the shortest proof but it contains in it a purely elementary proof which uses no representations theory at all [7]. Combining the links A, C, D gives of course a link from the Gerstenhaber Hesselink theorem to the Snapper conjecture, albeit a tenuous one. However, there is also a very direct link, due to Kraft [12] and this gives yet another proof of the Snapper conjecture.

One possible approach to the Snapper conjecture is of course via Young's rule (discussed below in section 6 ), which states that the irreducible representation [ $K$ ] occurs in $\rho(\lambda)$ with a multiplicity equal to the number of semistandard $k-t a b l e a u x$ of type $\lambda$. Indeed $i t$ is easy to show that the existence of a semistandard $k$-tableau of type $\lambda$ implies that $k<\lambda$. The inverse implication seems much more difficult to show directly. Yet this gives still another link between the Lidbler-Vitale theorem (Snapper conjecture) and the Gerstenhaber - Hesselink theorem. Both can be seen as consequences of the statement that there exists a semistandard $\lambda$-tableaux of type $\mu$ iff $\lambda<\mu$, cf. section 7.6 below. 


\section{YOUNG'S RULE AND THE SPECIALIZATION ORDER}

6.1. Young diagrams and semi-standard tableau. Let $k=\left(\kappa_{1}, \ldots, k_{m}\right)$ be a partition of $n$. As usual we picture $k$ as a Young diagram; that is an array of $n$ boxes arranged in $m$ rows with $k_{i}$ boxes in row $i$, as in the following example

$$
K=(4,3,3,2)
$$

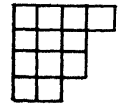

Let $\lambda=\left(\lambda_{1}, \ldots, \lambda_{s}\right)$ be another partition of $n$. Then a semistandard $k-t a b l e a u$ of type $\lambda$ is the Young diagram of $k$ with the boxes labelled by the integers $1, \ldots, s$ such that $i$ occurs $\lambda_{i}$ times, $i=1, \ldots, s$ and such that the labels are nondecreasing in each row of the diagram and strictly increasing along each column. An example of a $(5,3,2)$-tableau of type $(4,2,2,2)$ is

$$
\begin{array}{lllll}
1 & 1 & 1 & 1 & 4 \\
2 & 2 & 3 & & \\
3 & 4 & & &
\end{array}
$$

We shall use $n(k, \lambda)$ to denote the number of different semistandard $k$-tableau of type $\lambda$.

6.4. Young's rule. Let [o] denote the irreducible representation associated to the partition $\rho$. Then Young's rule (cf. [30]) says that

6.5. Theorem. Let $k$ and $\lambda$ be partitions of $\mathrm{n}$. Then the number of times that the irreducible representation $[\lambda]$ occurs in the permutation representation $\rho(k)$ is equal to the number of semistandard $\lambda$-tableaux of type $k$.

6.6. The specialization order and semistandard tableaux. The implication $k>\lambda \Leftarrow \rho(\lambda)$ is a direct summand of $\rho(k)$ follows easily from this. First, however, we state a lemma which is standard and seemingly unavoidable when dealing with the specialization order. Its proof is easy.

6.7. Lemma. Let $\lambda=\left(\lambda_{1}, \ldots, \lambda_{m}\right)$ and $k=\left(k_{1}, \ldots, k_{m}\right)$ be two partitions of $n$ and suppose that $\lambda>k$ and $(\lambda>\mu>k) \Rightarrow(\mu=\lambda$ or $\mu=k)$ for all partitions $\mu$. Then there are an $i$ and $a j, i<j$ such that $\kappa_{i}=\lambda_{i}+1, \lambda_{i}<\lambda_{i-1}, k_{j}=\lambda_{j}-1$, $\lambda_{j}>\lambda_{j+1}, k_{s}=\lambda_{s}, s \neq i, j$

Pictorially the situation looks as follows

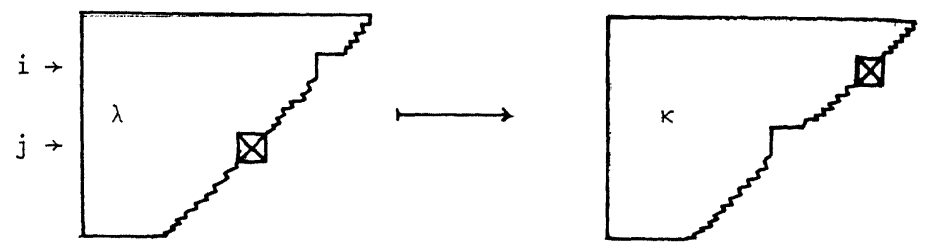


I.e. $a$ box in row $j$ which can be removed without upsetting \# (row $j$ ) $\geq$ \# (row $j+1$ ) (which means that we must have had $\lambda_{j}>\lambda_{j+1}$ ) is moved to a higher row $i$ which is such that it can receive it without upsetting \# (row $i$ ) $\leq$ \# (row $i-1$ ) (which means that we must have had $\lambda_{i}<\lambda_{i-1}$ ).

Of course not all transformations of the type described above result in a pair $\lambda, k$ such that there is no $\mu$ strictly between $\lambda$ and $\kappa$

6.8. Lemma. Let $\lambda$ and $k$ be two partitions of $n$ and suppose that there exists a semistandard $\lambda$-tableau of type $\kappa$. Then $\kappa>\lambda$.

Proof. In a semistandard $\lambda$-tableau of type $k$ all labels $i$ must occur in the first $i$ rows (because the labels in the columns must be strictly increasing). The number of labels $j$ with $j \leq i$ is $k_{1}+\ldots+k_{i}$ and the number of places available in the first $i$ rows is $\lambda_{1}+\ldots+\lambda_{i}$. Hence $\lambda_{1}+\ldots+\lambda_{i} \geq \kappa_{1}+\ldots+\kappa_{i}$ for all $i$ so that $\lambda<\kappa$.

6.9. The implication [K] occurs in $\rho(\lambda) \Rightarrow \kappa<\lambda$. Now suppose that [k] occurs in $\rho(\lambda)$. Then there is semistandard $k$-tableau of type $\lambda$ by Youngs rule so that $\kappa<\lambda$ by lemma 6.8 .

This implies of course that: $(\rho(\kappa)$ is a subrepresentation $\rho(\lambda)) \Rightarrow(\kappa<\lambda)$. Because there is obviously a semistandard $k$-tableau of type $k$ (in fact precisely one).

\section{NILPOTENT MATRICES AND SYSTEMS}

As was remarked in section 5 above the connection $A$ in the diagram above essentially consists of an almost identical proof of the two theorems. We start with a proof of the Gerstenhaber-Hesselink theorem. The first ingredient which we shall also need for the feedback orbits theorem is the following elementary remark on ranks of matrices.

7.1. Lemma. Let $A(t)$ be a family of matrices depending polynomially on a complex or real parameter $t$. Suppose that rank $A(t) \leq \operatorname{rank} A\left(t_{0}\right)$ for all $t$. Then rank $A(t)=\operatorname{rank} A\left(t_{0}\right)$ for all but finitely many $t$. This follows immediately from the fact that a polynomial in $t$ has only finitely many zeros.

7.2. Lemma. Let $\mathrm{A}$ be a nilpotent $\mathrm{n} \times \mathrm{n}$ matrix and let $\mathrm{F}$ be such that

$$
F\left(\operatorname{Ker} A^{i}\right) \subset \operatorname{Ker} A^{i-1}, i=1,2, \ldots, n
$$

Then $t A+(1-t) F$ is similar to $A$ for all but finitely many $t$.

Proof. We show first that

$$
\operatorname{Ker}(t A+(1-t) F)^{i} \supset \operatorname{Ker} A^{i}
$$

for all t. Indeed from (7.3) with $i=1$ we see that $F(\operatorname{Ker} A)=0$ and $i t$ follows 
that $(t A+(1-t) F)(\operatorname{Ker} A)=0$ which proves $(7.4)$ for $i=1$. Assume with induction that (7.4) holds for all $i<s$. Then

$$
\begin{aligned}
& (t A+(1-F))^{S} \operatorname{Ker} A^{s}=(t A+(1-t) F)^{s-1}(t A+(1-t) F) \operatorname{Ker} A^{s} \\
& C(t A+(1-t) F)^{s-1} \operatorname{Ker} A^{s-1}=0
\end{aligned}
$$

because $A \operatorname{Ker} A^{s} \subset \operatorname{Ker} A^{s-1}$ and $F \operatorname{Ker} A^{s} \subset \operatorname{Ker} A^{s-1}$ by (7.3). This proves (7.4). Using 7.4 we know by (7.1) that for almost all $t$ (take $t_{0}=1$ )

$$
\operatorname{rank}(t A+(1-t) F)^{i}=\operatorname{rank}\left(A^{i}\right)
$$

and because $t A+(1-t) F)$ and $A$ are both nilpotent it follows that $t A$ and $(1-t) F$ are similar for the all but finitely many $t$ for which (7.5) holds.

Now let $A$ be a nilpotent matrix. We say that $A$ is of type $k=\left(\kappa_{1}, \ldots, \kappa_{m}\right)$ if the Jordan normal form of A consists of m Jordan blocks of sizes $\kappa_{i} \times_{i}$, $i=1, \ldots, m$. E.g. A is of type $(4,2)$ iff its Jordan form is

$$
\left[\begin{array}{llll|ll}
0 & 1 & 0 & 0 & 0 & 0 \\
0 & 0 & 1 & 0 & 0 & 0 \\
0 & 0 & 0 & 1 & 0 & 0 \\
0 & 0 & 0 & 0 & 0 & 0 \\
\hline 0 & 0 & 0 & 0 & 0 & 1 \\
0 & 0 & 0 & 0 & 0 & 0
\end{array}\right]
$$

Consider $\operatorname{Ker} A, \operatorname{Ker} A^{2}, \ldots, \operatorname{Ker} A^{n}$. Then $A$ is of type $\kappa$ iff $\operatorname{dim}\left(\operatorname{Ker} A^{i}\right)=$ $\kappa_{1}^{*}+\ldots+k_{i}^{*}, i=1, \ldots, n$ where $k^{*}$ is the dual partition of $k$. Thus in the example the kernel spaces $\operatorname{Ker} A^{i}$ are spanned by the basis vectors $\left\{e_{1}, e_{5}\right\}$, $\left\{e_{1}, e_{2}, e_{5}, e_{6}\right\},\left\{e_{1}, e_{2}, e_{3}, e_{5}, e_{6}\right\},\left\{e_{1}, e_{2}, e_{3}, e_{4}, e_{5}, e_{6}\right\}$.

7.6. Semistandard tableaux and nilpotent matrices. Let $A$ be a nilpotent matrix of type $k$. Let $\mu$ be another partition of $n$ and suppose that there is a $\mu^{*}$-tableau of type $\kappa^{*}$. Then there is nilpotent matrix $F$ such that $F\left(\operatorname{Ker} A^{i}\right) \subset \operatorname{Ker} A^{i-1}$ for all $i$. This matrix $F$ is constructed as follows. First choose a basis $e_{1}, \ldots, e_{n}$ of $\mathbf{R}^{n}$ such that the first $k_{1}^{*}+\ldots+k_{1}^{*}$ elements of this basis form a basis for $\operatorname{Ker} A^{i}, i=1, \ldots, n$. Now consider a semistandard $\mu^{*-t a b l e a u} \mathrm{~T}$ of type $\kappa^{*}$. Take the Young-diagram of $\mu^{*}$ and label the boxes of it by the basis vectors $e_{1}, \ldots, e_{n}$ in such a way that the boxes marked with $i$ in the semistandard tableau $\mathrm{T}$ are filled with the basis vectors $e_{K_{1}^{*}+\ldots+K_{i-1}^{*}}, \ldots, e_{K_{1}^{*}+\ldots+K_{i}^{*}}$ This can be done because $T$ is of type $K^{*}$ so that theré are precisely $\kappa_{i}^{*}$ boxes labelled $i$ in $T$. Call this new $\mu^{*}$-tableau $T^{\prime}$. Now define $F$ by $F\left(e_{i}\right)=e_{i}$, if $e_{i}$, is just above $e_{i}$ in the $\mu *-t a b l e a u ~ T '$ and $F\left(e_{j}\right)=0$ if $e_{j}$ occurs in the first row of $T^{\prime}$. Then obviously 
$\operatorname{dim} \operatorname{Ker} F^{i}=\mu_{1}^{*}+\ldots+\mu_{i}^{*}$ so that $F$ is of type $\mu$ and $F\left(\operatorname{Ker} A^{i}\right) \subset \operatorname{Ker} A^{i-1}$ because the $\mu^{*-t a b l e a u} \mathrm{~T}$ was semistandard which implies that the labels are strictly increasing along columns.

An example may illustrate things. Let $\kappa^{*}=(2,2,2), \mu^{*}=(4,1,1)$. A $\mu^{*-t a b l e a u}$ of type $\kappa^{*}$ is then

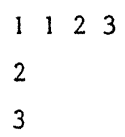

Inserting $e_{1}, \ldots, e_{6}$ in such a way that $e_{1}, e_{2}$ are put into boxes marked with $1, e_{3}, e_{4}$ in boxes marked with 2 and $e_{5}, e_{6}$ in boxes marked with 3 gives for example

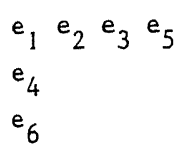

which yields an $F$ defined by $F\left(e_{6}\right)=e_{4}, F\left(e_{4}\right)=e_{1}, F\left(e_{1}\right)=F\left(e_{2}\right)=F\left(e_{3}\right)=$ $=F\left(e_{5}\right)=0$.

7.7. Proof of the Gerstenhaber-Hesselink theorem (Cf. 2.11 above). The implication $\Rightarrow$ is immediate. Indeed if $A_{t} \in O(K)$ converges to $A_{0} \in O(\lambda)$ as $t \rightarrow 0$ then $\operatorname{rank}\left(A_{t}^{i}\right) \geq \operatorname{rank}\left(A_{0}^{i}\right)$ for $\operatorname{small} t$ and all $i=1, \ldots, n$. Hence $\operatorname{dim}\left(\operatorname{Ker} A_{t}^{i} \leq \operatorname{dim}\left(\operatorname{Ker} A_{0}^{i}\right)\right.$ for $\operatorname{small} t$ so that $\kappa_{1}+\ldots+\kappa_{i} \leq \lambda_{1}+\ldots+\lambda_{i}$ for all $i$, hence $k^{*}>\lambda^{*}$ and $k<\lambda$. To prove the opposite implication it suffices to show this in case that $\kappa$ is obtained from $\lambda$ by a transformation of the type described in lemma 6.7. (Because if $\overline{O(k)} \supset O(\lambda)$ and $\overline{O(\lambda)} \supset O(\mu)$, then $\overline{O(K)} \supset \overline{O(\lambda)}$, and hence $\overline{O(\kappa)} \supset O(\mu)$ ). Then $\lambda^{*}$ is obtained from $\kappa^{*}$ by a similar transformation. We recall the picture
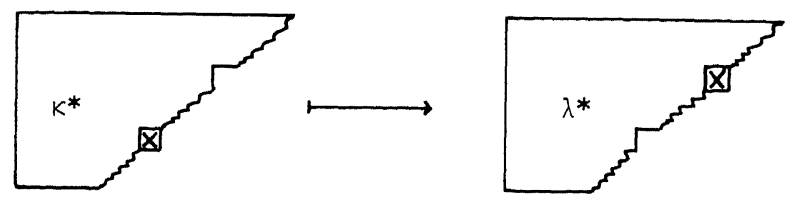

Now put $1^{\prime} s$ in the firstrow of $k^{*}, 2^{\prime} s$ in the second row, etc. Transport the box together with its label. The result is obviously a semistandard $\lambda^{*}$ tableau of type $\kappa^{*}$. Now let $A$ be a nilpotent matrix of type $\kappa$. Then by the construction of 7.6 above there is an $F$ of type $\lambda$ such that $F \operatorname{Ker} A^{i} \subset \operatorname{Ker} A^{i-1}$. Then $t A+(1-t) F$ is similar to $A$ for almost all $t$ by lemma 7.2 so that there is a sequence of $A^{\prime} s$ in $O(k)$ converging to $F \in O(\lambda)$, proving that $O(\lambda) \subset \overline{O(k)}$, which finishes the proof of the theorem.

Incidentally it is quite easy to describe $\mathrm{F}$ directly without ressorting to semistandard tableaux. 
7.10. Kronecker indices of systems. Let $(A, B) \in L_{m, n}^{c r}$ be a completely reachable pair of matrices. Recall that this means that the matrix $R(A, B)=\left(B: A B: \ldots: A^{n} B\right)$ has rank $n$. Recall that the Kronecker indices $\kappa(A, B)$ of the pair $(A, B)$ are defined as follows. Let for $i=1, \ldots, n$

$$
v_{i}(A, B)=\text { space spanned by the column vectors of } A^{j}, j=0,1, \ldots, i-1
$$

Let $d_{i}=\operatorname{dim} v_{i}(A, B), e_{i}=d_{i}-d_{i-1}, d_{0}=0$. Then $e_{i} \leq e_{i-1}, i=1, \ldots, n-1$, and $\kappa(A, B)$ is defined as the dual partition of $n$

$$
\kappa(A, B)=e(A, B) *
$$

where $e(A, B)=\left(e_{1}, \ldots, e_{n}\right)$.

The orbits of the feedback group (cf. 2.6 above) acting on $L_{m, n}^{c r}$ are precisely the subsets of $\mathrm{L}_{\mathrm{m}, \mathrm{n}}^{\mathrm{cr}}$ with constant $k(\mathrm{~A}, \mathrm{~B})$. Let $U(k)$ be this orbit. The "degeneration of systems theorem" now says

7.13. Theorem. $\overline{U(k)} \supset U(\lambda) \Leftrightarrow \kappa>\lambda$

Here follows a proof which is virtually identical with the proof of the Gerstenhaber-Hesselink theorem given above. First if $\left(A_{t}, B_{t}\right) \rightarrow\left(A_{0}, B_{0}\right)$ as $t \rightarrow 0,\left(A_{t}, B_{t}\right) \in U(K),\left(A_{0}, B_{0}\right) \in U(\lambda)$, then $\operatorname{rank}\left(A_{t}^{i-1} B_{t}: \cdots: A_{t} B_{t} B_{t} B_{t} \geq\right.$ $\operatorname{rank}\left(A_{0}^{i-1} B_{0}: \cdots: A_{0} B_{0} B_{0}\right)$ for small t. Hence $\operatorname{dim} v_{i}\left(A_{t}, B_{t}\right) \geq \operatorname{dim} v_{i}\left(A_{0}, B_{0}\right)$ for small $t$. Hence $e\left(A_{t}, B_{t}\right)<e\left(A_{0}, B_{0}\right)$ for small $t$ and $k\left(A_{t}, B_{t}\right)>k\left(A_{0}, B_{0}\right)$ for small $t$ which proves the implication $\Rightarrow$.

To prove the inverse implication it suffices to prove this in the case $\lambda$ is obtained from $k$ by a transformation as described in lemma 6.7 (exactly as in the case of the Gerstenhaber-Hesselink theorem). This means that $k *$ is obtained from $\lambda$ *by a similar transformation:
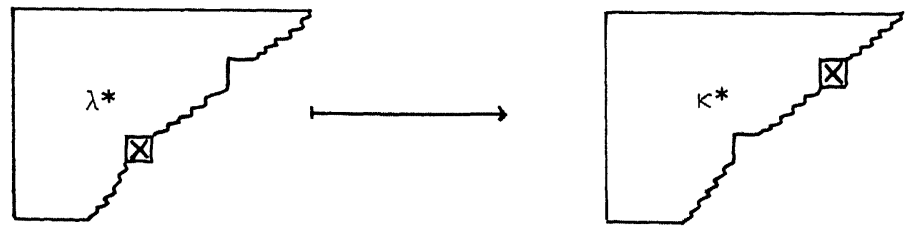

Now let $(A, B) \in U(K)$. Choose a basis $e_{1}, \ldots, e_{n}$ for $\mathbf{R}^{n}$ such that the first $k_{1}^{*}+\ldots+k_{i}^{*}$ elements of $e_{1}, \ldots, e_{n}$, form a basis for $v_{i}(A, B), i=1, \ldots, n$. Now write in the $e_{1}, \ldots, e_{n}$ in $k^{*} i_{n}$ the standard way and transport backwards together with its label. E.g. if $\lambda^{*}=(4,3,2,2,1)$ and $k^{*}=(4,4,2,1,1)$ then this would give 


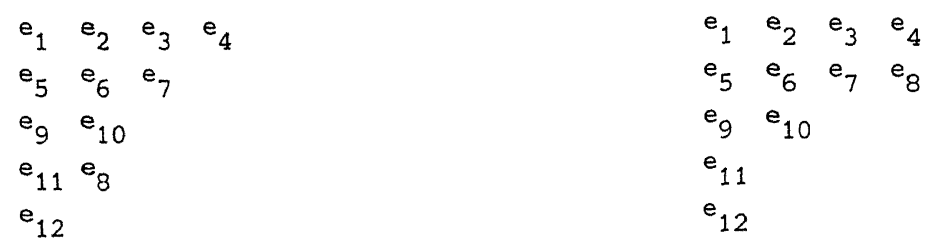

Then the vectors in the first $i$ rows of $k^{*}$ are a basis for $V_{i}(A, B)$. Now define a pair $(F, G)$ in terms of $\lambda^{*}$ as follows. G consists of the vectors in the first row of $\lambda^{*}$ (plus a zero vector in case $k_{1}^{*}>\lambda_{1}^{*}$ ), and $F$ is defined by $F\left(e_{i}\right)=e_{i}$, if $e_{i}$, occurs just below $e_{i}$ in $\lambda^{*}$ and $F\left(e_{i}\right)=0$ otherwise. Then $(F, G)$ has the following properties (all imediate)

$$
\begin{aligned}
& \text { (i) }(\mathrm{F}, G) \in U(\lambda) \subset L_{m, n}^{c r} \\
& \text { (ii) } \mathrm{V}_{i}(\mathrm{~F}, \mathrm{G}) \subset \mathrm{V}_{i}(\mathrm{~A}, \mathrm{~B}) \\
& \text { (iii) } \mathrm{FV}_{\mathrm{i}}(\mathrm{A}, \mathrm{B}) \subset \mathrm{V}_{i+1}(\mathrm{~A}, \mathrm{~B})
\end{aligned}
$$

(Of course (ii) follows from (iii) together with $V_{1}(F, G) \subset V_{1}(A, B)$ ). Now consider $A_{t}=t A+(1-t) F, B_{t}=t B+(1-t) G$. Then

$$
\begin{array}{ll}
v_{i}\left(A_{t}, B_{t}\right) \subset v_{i}(A, B) & \text { for all } t \\
v_{i}\left(A_{t}, B_{t}\right)=v_{i}(A, B) & \text { for all but finitely many } t
\end{array}
$$

Indeed obviously $v_{1}\left(A_{t}, B_{t}\right) \subset V_{1}(A, B)$ because of (ii) above for $i=1$. Now assume that $(7.14)$ holds for all $i<r$. Then

$$
\begin{aligned}
v_{r}\left(A_{t}, B_{t}\right) & =(t A+(1-t) F) V_{r-1}\left(A_{t}, B_{t}\right)+v_{r-1}\left(A_{t}, B_{t}\right) \\
& \subset t A V_{r-1}(A, B)+(1-t) F V_{r-1}(A, B)+v_{r-1}(A, B) \\
& \subset v_{r}(A, B)+v_{r}(A, B)+v_{r-1}(A, B)=v_{r}(A, B)
\end{aligned}
$$

This proves (7.14) and (7.15) follows by means of lemma 7.1 (with $t_{0}=1$ ) because

$$
\operatorname{dim} v_{i}\left(A_{t}, B_{t}\right)=\operatorname{rank}\left(A_{t}^{i-1} B_{t}, \ldots\left(B_{t}\right)\right.
$$

Now $\left(A_{t}, B_{t}\right) \rightarrow(F, G) \in U(\lambda)$ as $t \rightarrow 0$ and by (7.15) (and the theorem that the orbits under the feedback group are classified by the Kronecker indices) all but finitely many of the $\left(A_{t}, B_{t}\right)$ are feedback equivalent to $(A, B)$. Thus $U(\lambda) ;(F, G) \in \overline{U(K)}$ proving the theorem.

7.16. Remarks. The two proofs are very similar (up to duality in a certain sense). This can be given more precise form as follows. For a nilpotent matrix $N \in N_{n}$ let $\underline{s}(N)=\left\{(A, B) \in L_{m, n}^{c r} \mid N_{A}^{i} A^{i-1} B=0, i=1, \ldots, n\right\}$ and for 
$(A, B) \in L_{m, n}^{c r}$ let $\underline{t}(A, B)=\left\{N \in N_{n} \mid N^{i} A^{i-1} B=0, i=1, \ldots, n\right\}$. Then using the results above one shows that

$$
\underline{t} \underline{s}(\overline{O(\kappa)})=\overline{O(\kappa)}, \underline{s} \underline{t}(\overline{U(\kappa)})=\overline{U(\kappa)}
$$

so that $\underline{t}$ and $\underline{s}$ set up a bijective correspondence between the closures of orbits in the two cases and hence induce a bijective order preserving correspondence between the orbits themselves.

\section{VECTORBUNDLES AND SYSTEMS}

This section contains a modified version of the construction of MartinHermann [15] associating a vector bundle $E(\Sigma)$ over the Riemann sphere $\mathbb{P}^{1}(\mathbb{C})$ to every $\Sigma=(A, B) \in L_{m, n}^{c r}$. This version makes it almost trivial to see that $E(\Sigma)$ splits as a direct sum of line bundles $L\left(\kappa_{i}\right), i=1, \ldots, m$ where $k=\left(K_{1}, \ldots, K_{m}\right)$ is the set of Kronecker indices of $\Sigma$.

The first thing needed is some more information on the universal bundle $\xi_{\mathrm{m}}$.

8.1. On the universal bundle $\xi_{m} \rightarrow G_{n}\left(\mathbb{C}^{n+m}\right)$. Let $V$ be a complex $n+m$ dimensional vectorspace and $V^{*}=\operatorname{Hom}_{\mathbb{C}}(V, \mathbb{C})$ its dual vectorspace. Given $x \in G_{n}\left(\mathbb{C}^{n+m}\right)$ define $\mathrm{x}^{*}=\left\{\mathrm{v}^{*} \in \mathrm{V}^{*} \mid\left\langle\mathrm{v}^{*}, \mathrm{v}\right\rangle=0\right.$ for $\left.\mathrm{all} \mathrm{v} \in \mathrm{x}\right\}$ where $<$, $>$ denotes the usual pairing $\mathrm{V}^{*} \mathrm{x} \mathrm{V} \rightarrow \mathbb{C}$. Then $\mathrm{x}^{*}$ is $\mathrm{m}$-dimensional and $\mathrm{x} \mapsto \mathrm{x}^{*}$ defines a holomorphic isomorphism

$$
d: G_{n}(V) \rightarrow G_{m}\left(V^{*}\right)
$$

Now $v \in V / x$ defines a unique homomorphism $v^{T}: x^{*} \rightarrow \mathbb{C}$ as follows: $v^{T}(a)=\langle a, \tilde{v}\rangle$ for all a $\in x^{*}$, where $\tilde{v} \in V$ is any representant of $v$. This is well defined because $\langle a, b\rangle=0$ for $a l l b \in \mathrm{x}$ if $\mathrm{a} \in \mathrm{x}^{*}$. This defines an isomorphism between the pullback $\left(\mathrm{d}^{-1}\right)^{\prime} \xi_{\mathrm{m}}$ and the dual of the subbundle $\eta_{\mathrm{m}}$ of $G_{\mathrm{m}}\left(\mathrm{V}^{*}\right)$ defined by

$$
\eta_{\mathrm{m}}=\left\{\left(\mathrm{x}^{*}, \mathrm{w}\right) \in G_{\mathrm{m}}\left(\mathrm{V}^{*}\right) \times \mathrm{V}^{*} \mid \mathrm{w} \in \mathrm{x}^{*}\right\}
$$

It follows that $\xi_{m}$ is a subbundle of an $n+m$ dimensional trivial bundle $G_{n}\left(\mathbb{C}^{n+m}\right) \times \mathbb{C}^{n+m}$. Because $G_{n}\left(\mathbb{C}^{n+m}\right)$ is projective (compact) all holomorphic maps $G_{n}\left(\mathbb{C}^{n+m}\right) \rightarrow \mathbb{C}$ are constant so that the space of holomorphic sections $\Gamma\left(G_{n}\left(\mathbb{C}^{n+m}\right) \times \mathbb{d}^{n+m} \quad, G_{n}\left(\mathbb{C}^{n+m}\right)\right)$ is of dimension $n+m$. As a subbundle of a trivial $(n+m)$-dimensional bundle $\xi_{m}$ can therefore have at most $(n+m)$ linearly independent holomorphic sections. But we have already found $(n+m)$ linearly independent sections viz. the $\varepsilon_{1}, \ldots, \varepsilon_{n+m}$ defined by $\varepsilon_{i}(x)=e_{i} \bmod x$ where $e_{i}$ is the $i-t h$ standard basis vector of $\mathbb{a}^{n+m}$. Therefore

$$
\operatorname{dim} \Gamma\left(\xi_{m}, G_{n}\left(\mathbb{C}^{n+m}\right)\right)=n+m
$$

Now let $A \in G L_{n+m}(\mathbb{C})$. Then $A$ induces a holomorphic automorphism $A_{*}$ of 
$G_{(}\left(\mathbb{C}^{n+m}\right)$ defined by $x \mapsto A x$. Then of course there is an induced isomorphism $A^{-1}: \mathbb{c}^{n+m} / A x \rightarrow \mathbb{C}^{n+m} / x$ which for varying $x$ induces an isomorphism

$$
\mathrm{A}_{*}^{\prime} \xi_{\mathrm{m}} \simeq \xi_{\mathrm{m}}, A \in G L_{\mathrm{n}+\mathrm{m}}(\mathbb{C})
$$

8.5. The line bundles $L(i)$ over $\mathbb{P}^{1}(\mathbb{C})$. The Riemann sphere $\mathbb{P}^{1}(\mathbb{C})=s^{2}$ can be obtained by gluing together two copies of $\mathbb{C}$ along the open subsets $\mathbb{C} \backslash\{0\}$ by the isomorphism

$$
\mathbb{C} \backslash\{0\} \rightarrow \mathbb{C} \backslash\{0\}, s \mapsto t=\mathrm{s}^{-1}
$$

A line bundle over $\mathbb{P}^{1}(\mathbb{C})$ is then obtained by giving a holomorphic isomorphism $\mathbb{C} \backslash\{0\} \times \mathbb{C} \rightarrow \mathbb{C} \backslash\{0\} \times \mathbb{C}$ linear in the second variable compatible with the above isomorphism. Obviously the only possibilities are $(s, v) \mapsto\left(s^{-1}, s^{i} v\right)$ for $i \in \mathbb{Z}$. This gives us the following commutative diagram of identifications

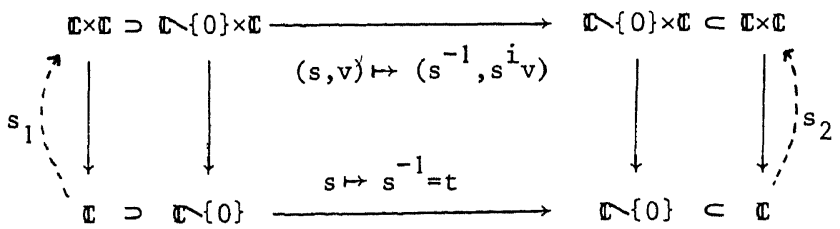

The corresponding holomorphic line bundle is denoted $L(-i)$. A section of $L(-i)$ consists of two holomorphic mappings $s_{1}, s_{2}$ of the form $s \mapsto(s, f(s))$, $t \mapsto(t, g(t))$ such that $s^{i} f(s)=g\left(s^{-1}\right)$. It readily follows that $f(s)$ must be a polynomial of degree $\leq-i$. Thus

$$
\begin{aligned}
& \operatorname{dim} \Gamma(L(i))=0 \text { if } i<0 \\
& \operatorname{dim} \Gamma(L(i))=i+1 \text { if } i \geq 0
\end{aligned}
$$

8.8. The (modified) Martin-Hermann vectorbundle of a system. Let $\Sigma=(A, B)$ be a pair of real or complex matrices of sizes $n \times n$ and $n \times m$. Then $(A, B)$ is completely reachable $(c r)$ iff the $n \times(n+m)$ matrix (sI - A B) is of rank $n$ for all complex values of $\mathrm{s}$. So if $\Sigma=(A, B)$ is $\mathrm{cr}$ one can define a holomorphic map $\psi_{\Sigma}$ by

$$
\psi_{\Sigma}: \mathbf{P}^{1}(\mathbb{E}) \rightarrow G_{n}\left(\mathbb{C}^{n+m}\right), s \mapsto \operatorname{Row}(s I-A ; B), \infty \mapsto \operatorname{Row}(I ; 0)
$$

where Row(M) for an $n \times(m+n)$ matrix $M$ denotes the subspace of $\mathbb{C}^{n+m}$ generated by the rows of $M$. The vectorbundle $E(\Sigma)$ over $\mathbb{P}^{1}(\mathbb{C})$ is now defined by

$$
E(\Sigma)=\psi_{\Sigma}^{\prime} \xi_{m}
$$

8.11. Proposition. $E(\Sigma)$ depends only on the feedback orbit of $\Sigma$. Indeed one easily checks that $\Sigma=(A, B), \Sigma^{\prime}=\left(A^{\prime}, B^{\prime}\right) \in L_{m, n}^{c r}$ are feedback 
equivalent (cf. 2.6. above) iff there are constant invertible matrices $P, Q$ such that $P(s I-A ! B) Q=\left(s I-A^{\prime}: B^{\prime}\right)$. Now Row $(P M)=\operatorname{Row}(M)$ and postmultiplication with $Q$ changes $\psi_{\Sigma}$ to $Q_{*} \circ \psi_{\Sigma}$ and $E\left(\Sigma^{\prime}\right)=\psi_{\Sigma}^{!},\left(\xi_{\mathrm{m}}\right)=\psi_{\Sigma}{ }_{\Sigma}^{\prime}\left(Q_{*}^{\prime}{ }^{\prime}{ }_{\mathrm{m}}\right) \simeq \psi_{\Sigma}^{\prime}\left(\xi_{\mathrm{m}}\right)=\mathrm{E}(\Sigma)$ by 8.4 above, proving the proposition.

Thus to determine $E(B)$ we can assume that $\Sigma=(A, B)$ is in Brunowsky canonical form which means that $A, B$ take the form

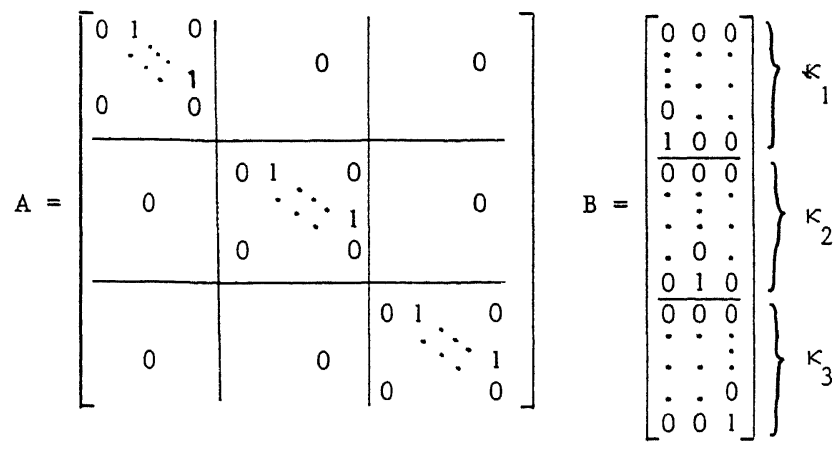

in case $m=3$, where $\left(K_{1}, K_{2}, K_{3}\right)=\kappa(A, B)$ are the kronecker indices of $\Sigma=(A, B)$. (The general case is evident from this example). The matrix (sT - A B) is now easily written down, and one observes that for all $s \neq 0, \infty \quad e_{1} \equiv e_{2} \equiv \ldots \equiv e_{K} \equiv e_{n+1}$ $\bmod \operatorname{Row}\left(\mathrm{sT}-\mathrm{A}:\right.$ B), i.e. $\bmod \psi_{\Sigma}(\mathrm{s})$ and for $\mathrm{s}=0, e_{2} \equiv \ldots \equiv e_{K} \equiv e_{n+1} \equiv 0$ but $e_{1} \not 0$ and for $s=\infty, e_{1} \equiv \ldots \equiv e_{k} \equiv 0$ and $e_{n+1} \not 0$. It follows that the vectors $\varepsilon_{1}\left(\psi_{\Sigma}(s), \ldots, \varepsilon_{K_{1}}\left(\psi_{\Sigma}(s)\right), \varepsilon_{n+1}\left(\psi_{\Sigma}(s)\right)\right.$ span a one dimensional subspace of $\xi_{m}\left(\psi_{\Sigma}(s)\right)$ for all s so that $E(\Sigma) \simeq \psi_{\Sigma}^{!} \xi_{\mathrm{m}}$ contains a line bundle $\mathrm{L}_{1}$ which admits at least $k_{1}+1$ linearly independent holomorphic sections viz. the $\varepsilon_{1}, \ldots, \varepsilon_{K_{1}}, \varepsilon_{n+1}$. Similar relations hold for $\varepsilon_{\kappa_{1}+\ldots+\kappa_{i-1}+1}, \ldots, \varepsilon_{\kappa_{1}+\ldots+\kappa_{j}}, \varepsilon_{n+i}$ for all $1=1, \ldots, m$ giving us subbundles ${ }^{L_{i}}, i=1, \ldots$, m which admi $i^{i}$ at least $k_{i}+1$ holomorphic sections. This exhausts the $\varepsilon_{j}$ and because the $\varepsilon_{1}(x), \ldots, \varepsilon_{n+m}(x)$ span $\xi_{m}(x)$ for a11 $x \in G_{n}\left(C / I^{n+m}\right)$ it follows that $E(\Sigma)=\oplus L_{i}$. As the pullback or a bundle $\xi_{m}, E(\Sigma)$ itself is a subbundle of an $(n+m)$-dimensional trivial bundle. Because $\mathbb{P}(\mathbb{C})$ is projective it follows (as before) that $E(\Sigma)$ has at most $n+m$ linearly independent holomorphic sections. But $\mathrm{L}_{i}$ has at least $k_{i}+1$ linearly independent sections, hence $\oplus \mathrm{L}_{i}$ has at least $\Sigma\left(\kappa_{i}+1\right)=n+m$ linearly independent sections which proves that $L_{i}$ has precisely $\kappa_{i}+1$ linearly independent sections and hence identifies $L_{i}$ as the bundle $L\left(\kappa_{i}\right)$ described above in $(8.5)$. We have reproved the theorem of Martin and Hermann [15]

8.12. Theorem. Keeping the notations introduced above in (8.10) and (8.5) we have $E(\Sigma) \simeq \underset{i=1}{\oplus} L\left(K_{i}\right)$.

8.13. The correspondence B. (cf. the diagram in section 5 above). The mapping 
$\Sigma \mapsto E(\Sigma)$ is obviously continuous. Thus the result $U(\kappa) \supset U(\lambda) \Leftrightarrow \kappa>\lambda$ can be deduced from Shatz's theorem (cf. 2.9). Inversely Shatz's theorem for positive bundles over $\mathbb{P}^{1}(\mathbb{C})$ can be deduced from the result on feedback orbits because every positive bundle arises as an $E(\Sigma)$. By tensoring with a suitable $L(r)$, $r$ high enough, the result is then extended to arbitrary bundles over $\mathbb{P}^{1}(\mathbb{C})$.

\section{VECTOR BUNDLES, SYSTEMS AND SCHUBERT CELLS}

9.1. Partitions and Schubert-cells. Let $k$ be a partition of $n$. To $k$ we associate the following increasing sequence of $n$ numbers $\tau(k)$.

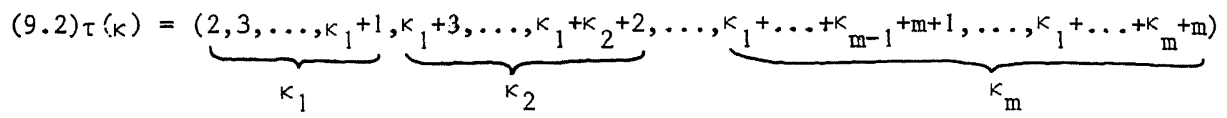

Let $\tau_{j}(k), j=1, \ldots, n$ be the $j$-th element of this sequence. It is an easy exercise to check that

$$
\kappa>\lambda \Leftrightarrow \tau_{i}(k) \geq \tau_{i}(\lambda) \text { for all } i=1, \ldots, n
$$

Thus the specialization order is a suborder of the inclusion ordening between closed Schubert cells, because $\operatorname{SC}(\tau) \supset \operatorname{SC}\left(\tau^{\prime}\right) \Leftrightarrow \tau_{i} \geq \tau_{i}^{\prime}, i=1, \ldots, n$. And in turn as we saw above in section 4 the Schubert-cell order is a quotient of the BGG order on the Wey 1 group $\mathrm{S}_{\mathrm{n}+\mathrm{m}}$.

9.4. Systems and Schubert cells. Let $(A, B) \in L_{m, n}^{c r}$ be a system and as in section 8.8 consider the associated holomorphic morphisn $\psi_{\Sigma}: \mathbb{P}^{1}(\mathbb{C}) \rightarrow G_{n}\left(\mathbb{C}^{n+m}\right)$. Suppose that $(A, B)$ are in Brunovsky canonial form. Then simple inspection of the matrix (sT-A;B) (cf. the example below proposition 8.11) shows that Im $\psi_{E} \subset \operatorname{SC}(\tau(K))$, where $k=\kappa(A, B)$. Now let $(A, B)$ be any system in $L_{m, n}^{c r}$. Then it is feedback equivalent to a Brunovsky canonical one so that $(s T-A ; B)=P\left(s T-A_{0} ; B_{0}\right) Q$ for certain constant invertible matrices $P, Q$ where $\left(\mathrm{A}_{0}, \mathrm{~B}_{0}\right)$ is a canonical pair. Premultiplication with $\mathrm{P}$ does not change $\psi_{\Sigma}$ and postmultiplication with $Q$ induces an automorphism of $G_{n}\left(\mathbb{C}^{n+m}\right)$ which takes the "standard basis" Schubert-cell SC( $\tau(\kappa))$ into another Schubert-cell of the same dimension type. Thus we have shown.

9.5. Theorem. Let $\Sigma \in L_{m, n}^{c r}, k=k(\Sigma)$ and let $\psi_{\Sigma}: \mathbb{P}^{1}(\mathbb{C}) \rightarrow G_{n}\left(\mathbb{C}^{n+m}\right)$ be the Martin-Hermann morphism of $\Sigma$. Then there is a Schubert-cell SC( $\underline{A}), \underline{A}=\left(A_{1}, \ldots, A_{n}\right)$ such that $\operatorname{Im} \psi_{\Sigma} \subset S C(\underline{A})$ and $\operatorname{dim} A_{i}=\tau_{i}(\kappa)$, where $\tau_{i}(\kappa)$ is defined by (9.2).

The converse is also true but considerably more difficult to prove, cf.[8]:

9.6. Theorem. With the notations of theorem 9.5 let the Schubert-cell SC(B)), $\underline{B}=\left(B_{1}, \ldots, B_{n}\right)$, in $G_{n}\left(\mathbb{C}^{n+m}\right)$ be such that $\operatorname{Im} \psi_{\Sigma} \subset \operatorname{SC}(\underline{B})$. Then $\operatorname{dim} B_{i} \geq \tau_{i}(k)$.

9.7. Vectorbundles and Schubert cells. Because every positive vectorbundle 
over $\mathbf{P}^{1}(\mathbb{C})$ arises as the bundle $E(\Sigma)$ of some system $\Sigma$ one has the obvious analogues of theorems 9.5 and 9.6 for positive bundles over $\mathbf{P}^{1}(\mathbb{C})$. Here the morphism $\psi_{\Sigma}$ must of course be replaced by the classifying morphism (cf. section 3.2 above) of a positive vector bundle $\mathrm{E}$, and $\mathrm{n}+\mathrm{m}$ and $\mathrm{m}$ are determined respectively as $\operatorname{dim} \Gamma\left(E, \mathbb{P}^{1}(\mathbb{C})\right)$ and $\operatorname{dim} E$.

$$
\text { 10. A FAMILY OF REPRESENTATIONS OF } s_{n+m} \text { PARAMETRIZED BY } G_{n}\left(\mathbb{c}^{n+m}\right)
$$

10.1. Construction of the family. Let $M$ be the regular representation of $\mathrm{S}_{\mathrm{n}+\mathrm{m}}$. That is $M$ has a basis $e_{\sigma}, \sigma \in \mathrm{S}_{\mathrm{n}+\mathrm{m}}$ and $\tau \in \mathrm{S}_{\mathrm{n}+\mathrm{m}}$ acts by $\tau\left(\mathrm{e}_{\sigma}\right)=\mathrm{e}_{\tau \sigma^{\circ}}$. Now consider the universal bundle $\xi_{m}$ over $G_{n}\left(\mathbb{C}^{n+m}\right)$ and the $(n+m)$ holomorphic sections $\varepsilon_{1}, \ldots \varepsilon_{n+m}$ defined by $\varepsilon_{i}(x)=e_{i} \bmod x \in \mathbb{c}^{n+m} / x$. Take the $(m+n)$-fold tensor product of $\xi_{m}$ and define a family of homomorphisms parametrized by $G_{n}\left(\mathbb{c}^{n+m}\right)$ by

$$
\begin{aligned}
\pi_{x}: M & \rightarrow \xi_{m}(x)^{\theta(n+m)} \\
e_{\sigma}^{-1} & \mapsto \varepsilon_{\sigma(1)}(x) \otimes \ldots \otimes \varepsilon_{\sigma(n+m)}(x)
\end{aligned}
$$

(More precisely (10.2) defines a homomorphism of vectorbundles $\left.G_{n}\left(\mathbb{c}^{n+m}\right) \times M \rightarrow \xi_{m}^{\theta(n+m)}\right)$.

The group $s_{n+m}$ acts on $\xi_{m}(x)^{\theta(n+m)}$ by permuting the factors and it is a simple exercise to see that $\pi_{x}$ is equivariantwith respect to this action, i.e. that $\pi_{x}(\tau v)=\tau \pi_{x}(v)$ for all $v \in M$, $\tau \in S_{n+m}$ (Here the product $\tau \sigma \in S_{n+m}$ is interpreted as first the automorphism $\sigma$ of $\{1, \ldots, n+m\}$ and then the automorphism $\tau$ ).

Thus $\operatorname{Im} \pi_{x}=\pi(x)$ is a representation of $S_{n+m}$ for all $x$ giving us a family of representations parametrized by $G_{n}\left(\mathbb{C}^{n+m}\right)$. Fixing a point $x_{0} \in G_{n}\left(\mathbb{C}^{n+m}\right)$ and choosing $\mathrm{m}$ independent sections of $\xi_{\mathrm{m}}$ in a neighbourhood $U$ of $\mathrm{x}_{0}$, this gives us families of homomorphisms of representations

$$
M \stackrel{\pi_{x}^{\prime}}{\longrightarrow}\left(\mathbb{C}^{m}\right)^{\otimes(n+m)}, x \in U \subset G_{n}\left(\mathbb{C}^{n+m}\right)
$$

such that $\operatorname{Im} \pi_{x}^{\prime} \simeq \pi(x)$ for $x \in U$.

10.4. Permutation representations and Schubert-cells (On connection D). Let $x \in G_{n}\left(\mathbb{c}^{n+m}\right)$ be a subspace of $x^{n+m}$ spanned by the rows of a matrix of the form $(m=3, n=5)$

$$
\left[\begin{array}{llll|lll|l}
* & * & 0 & 0 & 0 & 0 & 0 & 0 \\
0 & * & * & 0 & 0 & 0 & 0 & 0 \\
0 & 0 & * & * & 0 & 0 & 0 & 0 \\
\hline 0 & 0 & 0 & 0 & * & * & 0 & 0 \\
0 & 0 & 0 & 0 & 0 & * & * & 0
\end{array}\right]
$$


where all the *'s are nonzero. Then obviously the representation $\pi(x)$ of $s_{8}$ is isomorphic to $\rho(\tilde{K})$ with $\tilde{K}=(4,3,1)$. Note that $x$ is in the standard Schubertcell $\operatorname{SC}(\tau(\kappa))$, with $K=(3,2,0)$. This holds in general and it is not difficult to extend this to

10.5. Proposition. Let $k$ be an m-part partition of $n, \tilde{k}=\left(\kappa_{1}+1, \ldots, k_{m}+1\right)$. Then for almost all $x \in \operatorname{SC}(\tau(k))$, the representation $\pi(x)$ of $S_{n+m}$ contains the representation $\rho(\tilde{k})$ and for some $x \in \operatorname{SC}(\tau(k)) \pi(x) \simeq \rho(\tilde{\kappa})$.

Conjecturally the reverse holds also. That is if for almost all $x$ in a standard Schulbert-cell SC $(\lambda)$ we have that $\pi(x)$ contains $\rho(\tilde{\kappa})$ then $\lambda_{i} \geq \tau_{i}(k)$, $i=1, \ldots, n$. And $I$ am even inclined to believe that if $x \in \operatorname{SC}(\lambda)$ and $\pi(x)$ contains (or is equal to) $\rho(\tilde{k})$ then $\lambda_{i} \geq \tau_{i}(k)$.

Note also that the matrices (10.5) are precisely the type of matrices $(s I-A \mid B)$ for a system $\Sigma=(A, B)$ in feedback canonical form $(s \neq 0, \infty)$, suggesting that there is a natural representation of $S_{n+m}$ attached to $\Sigma$ awaiting interpretation.

\section{DEFORMATIONS OF REPRESENTATION HOMOMORPHISMS AND SUBREPRESENTATIONS}

11.1. On proving Snapper-type results. Suppose we have given a continuous family of homomorphisms of finite dimensional representations over $\mathbb{C}$ of a finite group G

$$
\pi_{t}: M \rightarrow V
$$

Suppose that Im $\pi_{t} \simeq \rho$ for $t \neq 0$ (and sma11) and $\operatorname{Im} \pi_{0} \simeq \rho_{0}$. Then the representation $\rho_{0}$ is a direct summand of $\rho$. This is seen as follows. Because the category of finite dimensional representations of $G$ is semisimple there is a homomorphism of representations $\phi_{0}: \operatorname{Im} \pi_{0} \rightarrow M$ such that $\pi_{0} \circ \phi_{0}=$ id. Then $\pi_{t} \circ \phi_{0}: \operatorname{Im} \pi_{0} \rightarrow \operatorname{Im} \pi_{t}$ is still injective for small $t$ (by the continuity of $\pi_{t}$ ) which gives us $\rho_{0}$ as a subrepresentation and hence a direct summand of $\rho$.

It is almost equally easy to construct a surjective homomorphism $\operatorname{Im} \pi_{t}+\operatorname{Im} \pi_{0}$ (which is more or less what we shall do below in 11.3 in (a sketch of) a proof of the Liebler-Vitale theorem that $\kappa<\lambda \Rightarrow \rho(\kappa)$ is a direct summand of $\rho(\lambda)$ ).

11.2. The inverse result. Inversely if $\rho_{0}$ is a subrepresentation of $\rho$ then there is a family of representations (11.2) such that $\operatorname{Im} \pi_{t} \simeq \rho$ for $t \neq 0$ and Im $\pi_{0} \simeq p_{0}$, and if $\rho$ is generated (as a $\mathbb{C}[G]$-module) by one element one can take for $M$ in (11.2) the regular representation. Indeed if $\rho_{0}$ is a subrepresentation of $\rho$ then $\rho=\rho_{0} \oplus \rho_{1}$. Let $\pi: M \rightarrow \rho=\rho_{0} \oplus \rho_{1}$ be a surjective map of representations Let $\pi_{0}, \pi_{1}$ be the two components of $\pi$. Let $s=\left(s_{0}, s_{1}\right)$ be a section of $\pi$. Then $\pi_{0} s_{0}=i d, \pi_{1} s_{1}=i d, \pi_{0} s_{1}=0, \pi_{1} s_{0}=0$ and it follows that $\pi(t)$ 
consisting of the components $\pi_{0}$ and $t \pi_{1}$ is still surjective. Hence Im $\pi(t)=\rho$ and $\operatorname{Im} \pi(0)=\rho_{0}$.

11.3. On a proof of the Liebler-Vitale theorem. It is quite conceivable that the grand family constructed in section 10 above contains all subfamilies needed to prove the Liebler-Vitale theorem by means of the deformation argument of section 11.1 above. So far, however, we have not found them. A somewhat more complicated argument immediately suggested by the structure of the family of representations constructed in section 10 above does give a proof. It is perhaps best illustrated by means of an example.

Consider an $x \in G_{3}\left(\mathbb{C}^{5}\right)$ spanned by the rows of a matrix of the form

$$
\left[\begin{array}{rrrrr}
1 & -1 & 0 & 0 & 0 \\
0 & 1 & -1 & 0 & 0 \\
z & 0 & 0 & -1 & t
\end{array}\right]
$$

Let $f_{1}, \ldots, f_{5}$ be the images of the standard basis vectors $e_{1}, \ldots, e_{5}$ in $\mathbb{C}^{5} / x$. Then $f_{1}=f_{2}, f_{2}=f_{3}, f_{4}=z f_{1}+t f_{5}$ so that $f_{1}$ and $f_{5}$ are a basis for $\mathbb{C}^{5} / x$ for all values of $z$ and $t$. Let $(1) \in S_{5}$ be the identity representation. The image of e $(1) \in M$ in $\left(\mathbb{C}^{5} / x\right)^{85}$ is by the definition (10.2) equal to $(11.4) \quad f_{1} \otimes f_{2} \otimes f_{3} \otimes f_{4} \otimes f_{5}=z f_{11115}+t f_{11155}$

where $f_{11115}$ is short for $f_{1} \otimes f_{1} \otimes f_{1} \otimes f_{1} \otimes f_{5}$ and similarly for other 5tuples of indices. Symmetrizing the element (11.4) with respect to the permutation (45) gives us

$$
z\left(f_{11115}+f_{11151}\right)+2 t f_{11155}
$$

Let $V_{1}$ be the subrepresentation of Im $\pi_{x}$ (generated by the element (11.5). (The representation Im $\pi_{x}$ is the subrepresentation of $\left(c^{5} / x\right)^{85}$ generated by $(11.4))$. Now (11.5) is invariant under the Young subgroup $\mathrm{s}_{3} \times \mathrm{S}_{2}$. Hence $\operatorname{dim} v_{1} \leq 5 ! / 3 ! 2 !$. On the other hand if $t \neq 0$ then setting $z=0$ in (11.5) (which corresponds to the surjective map mentioned just above 11.2 associated to a family of representations) obviously maps $v_{1}$ onto the vector space with as basis all symbols $f \ldots$ with three of the indices equal to 1 and 2 equal to 5 . This is $\rho(3,2)$ of dimension $5 ! / 3 ! 2 !$ so that $v_{1} \simeq \rho(3,2)$ if $t \neq 0$. Now for $z \neq 0$ set $t=0$ in $(11.4)$ to obtain a homomorphism of representations

$$
\operatorname{Im} \pi_{x} \rightarrow \rho(4,1)
$$

It is now not hard to prove that (cf. [7] for a detailed proof)

11.6. Proposition. The composed homomorphism of representations $\rho(3,2) \simeq \mathrm{V}_{1} \subset \operatorname{Im} \pi_{\mathrm{x}} \rightarrow \rho(4,1)$ is surjective.

This then proves that $\rho(4,1)$ is a direct summand of $\rho(3,2)$. The argument 
generalizes without difficulty for partitions $k>\lambda$ such that $\lambda$ is obtained from $k$ by a transformation of the type described in 6.7 above.

This is by no means the easiest way to prove the Liebler-Vitale theorem. It is perfectly easy to describe the morphism $\rho(K) \rightarrow \rho(\lambda)$ directly and then the general analogue of proposition 11.6 yields the Liebler-Vitale result. This proof uses no representation theory at all (except the definition of the permutation representations $\rho(k)$ ); cf. [7] for details.

\section{References}

1.I.N. Bernstein, I.M. Gelfand, S.I. Gelfand, Schubert cells and cohomology of the spaces G/P, Russian Math. Surveys 28, 3 (1973), 1-26.

2.T. Brylawski, The lattice of integer partitions, Discrete Math. 6 (1973), 201-219.

3.M. Gerstenhaber, On dominance and varieties of commuting matrices, Ann.of Math. 73 (1961), 324-348.

4.A. Grothendieck, Sur la classification des fibrés holomorphes sur la sphère de Riemann, Amer.J.Math. 79 (1957), 121-138.

5.L. Harper, G.-C. Rota, Matching theory: an introduction, In: P. Ney (ed.), Advances in Probability Vol. 1, Marcel Dekker, 1971, 171-215.

7.M. Hazewinke1, T. Vorst, on the Snapper, Liebler-Vitale, Lam theorem on permutation representations of the symmetric groups, in preparation.

8.M. Hazewinkel, C. Martin, Representations of the symmetric group, the specialization order, systems and Grassmann manifolds, in preparation.

9.W. Hesselink, Singularities in the nilpotent scheme of a classical group, Trans. Amer.Math.Soc. 222 (1976), 1-32.

10R.E. Kalman, Kronecker invariants and feedback (+ errata), In: L. Weiss (ed.) Ordinary differential equations, Acad.Pr., 1972, 459-471.

11.A. Kerbor, The diagram lattice as structural principle in mathematics. In: P. Kramers, A. Rieckers (eds.), Group theoretical methods in physics (Tübingen, 1977), Lect.Notes in Physics 79, Springer, 1978, 53-71.

12.H.P. Kraft, Letter to M. Hazewinke1, June 2, 1980 (This material will be presented at the Torun conference in Sept. 1980).

13.T.Y. Lam, Young diagrams, Schur functions, the Gale Ryser theorem and a conjecture of Snapper, J. pure and appl.Algebra 10 (1977), 81-94.

14.R.A. Liebler, M.R. Vitale, ordening the partition characters of the symmetric group, J. of Algebra 25, (1973), 487-489.

15.C. Martin, R. Hermann, Applications of algebraic geometry to system theory: the McMillan degree and Kronecker indices of transfer functions as topological an holomorphic system invariants, SIAM J. Control Opt. 16 (1978), 743-755.

16.A. Mead, E. Ruch, A. Schönhofer, Theory of chirality functions, generalized for molecules with chiral ligands, Theor.Chim.Acta 29 (1973), 269-304. 
17. J. Milner, J. Stasheff, Characteristic classes, Princeton Univ.Pr., 1974.

18.E. Ruch, A. Schönhofer, Theorie der Chiralitätsfunktionen, Theor.Chim.Acta 19 (1970), 225-287.

19.H. Ryser, Combinatorial mathematics, Carus Math. Monographs 14, Wiley, 1963.

20.S.S. Shatz, The decomposition and specialization of algebraic families of vector bundles, Compositio Math. 35 (1977).

21.E. Snapper, Group characters and nonnegative integral matrices, J.of Algebra $19(1971), 520-535$.

22.R.F. Muirhead, Some methods applicable to identities and inequalities of symmetric algebraic functions of $\mathrm{n}$ letters, Roc. Edinburgh Math.Soc. 21 (1903), 144-157.

23.M. Hazewinke1, A partial survey of the uses of algebraic geometry in systems and control theory, Proc. INDAM Vol. 24 (Severi centennial conference, Rome April 1979), Acad.Press, to appear.

24. M. Hazewinkel, (Fine) moduli spaces in linear system theory: what they are and what they are good for. In C. Martin, C. Byrnes (eds.), Algebraic and geometric methods in linear systems theory, Reidel Pub1.Cy, to appear.

25.J.E. Humphreys, Linear algebraic group, Springer, 1975.

26.J.C. Jantzen, Moduln met einem höchsten Gewicht, Lect.Notes in Math. 750, Springer, 1979.

27.J.E. Humphreys, Highest weight modules for semi-simple Lie algebras, In: Proc. Workshop on present trends in representation theory, Carleton Univ., 1979, Lect.Notes Math., Springer, to appear.

28.A.J. Coleman, Induced representations with applications to $S_{n}$, Queen's papers in pure and applied math. 4, Queen's Univ., Kingston, Canada, 1966.

29.C.W. Curtis, I. Reiner, Representation theory of finite group and associative algebras, Interscience, 1962 .

30.G.D. James, The representation theory of the symmetric groups, Lect. Notes in Math. 682, Springer, 1978 . 